\title{
Disulfiram Enhances the Activity of Polymyxin B Against Klebsiella pneumoniae by Inhibiting Lipid A Modification
}

\author{
Wei Huang $\mathbb{D}^{1,2, *}$, Jinyong Zhang $\mathbb{D}^{3, *}$, Shiyi Liu $\mathbb{D}^{\prime}$, Chunxia Hu', Min Zhang', Shumin Cheng', \\ Huijuan $\mathrm{Yu}^{1,2}$, Manling Zheng $\mathbb{D}^{1}$, Jinsong $\mathrm{Wu}^{2}$, Yuemei $\mathrm{Lu}^{2}$, Quanming Zou ${ }^{3}$, Ruiqin Cui ${ }^{1}$ \\ 'Bacteriology \& Antibacterial Resistance Surveillance Laboratory, Shenzhen Institute of Respiratory Diseases, Shenzhen People's Hospital (The Second \\ Clinical Medical College, Jinan University, The First Affiliated Hospital, Southern University of Science and Technology), Shenzhen, People's Republic of \\ China; ${ }^{2}$ Department of Clinical Microbiology, Shenzhen People's Hospital (The Second Clinical Medical College, Jinan University, The First Affiliated \\ Hospital, Southern University of Science and Technology), Shenzhen, People's Republic of China; ${ }^{3}$ National Engineering Research Center of \\ Immunological Products, Department of Microbiology and Biochemical Pharmacy, College of Pharmacy, Third Military Medical University, Chongqing, \\ People's Republic of China
}

*These authors contributed equally to this work

Correspondence: Quanming Zou; Ruiqin Cui, Email qmzou2007@I63.com; screening_sz@I63.com

Background: The use of antibiotic adjuvants is a complementary strategy to the development of new antibiotics. The essential role of the ArnA dehydrogenase domain (ArnA_DH) in the addition of 4-amino-L-arabinose (L-Ara4N) to lipid A makes it a potential target in polymyxin adjuvant design.

Purpose: This study aimed to identify a dehydrogenase inhibitor that enhances the antibacterial effect of polymyxin B (PB) and to further understand the mechanism of this drug combination.

Methods: A susceptible K. pneumoniae strain, ATCC13883, was used to screen a dehydrogenase inhibitor library based on 3-(4,5)dimethylthiazol(-z-y1)-2,5-diphenyltetrazolium bromide (MTT) and chequerboard assays. The protein- and cell-based effects of disulfiram (DSF) on ArnA activity were assessed, and the transcription levels of genes in the arn operon were evaluated by quantitative real-time polymerase chain reaction (qRT-PCR). Lipid A was isolated, and a structural analysis was performed. The cell wall function was evaluated through membrane integrity and bacterial viability assays. The in vivo antibacterial activity was evaluated using a mouse pulmonary infection model.

Results: We screened a dehydrogenase inhibitor library and found that the anti-alcoholism drug DSF significantly enhanced the antibacterial activity of PB in vitro and in vivo. The protein-based enzyme activity assay showed that DSF exerted no direct effect on the dehydrogenase activity of ArnA. Treatment with the combination of DSF and PB but not with PB alone decreased both the transcription level of genes in the arn operon and the modification level of lipid A. DSF also strengthened the disruption of the cell membrane integrity of PB. Moreover, the enhanced PB antibacterial activity was effective against clinical PB-resistant strains.

Conclusion: We identified a new drug combination that can be used to reduce the necessary dosage of $\mathrm{PB}$ and overcome $\mathrm{PB}$ resistance, and this drug combination has good prospects for clinical application.

Keywords: polymyxins, antibiotic adjuvants, antibacterial, cell membrane

\section{Introduction}

Due to the spread of antibiotic resistance and a lack of antibiotics effective against gram-negative organisms, colistin and polymyxin B (PB), members of the polymyxin class of antimicrobial peptides, have seen increased clinical use as drugs of last resort. ${ }^{1,2}$ Although they exhibit efficacy against nearly all gram-negative bacteria, these peptides can cause irreversible kidney damage when administered at therapeutic doses. ${ }^{3,4}$ Moreover, resistance to these antibiotics is increasingly being found among clinical isolates. ${ }^{5,6}$ Klebsiella pneumoniae is one of the main pathogens of nosocomial infection and has developed a wide range of resistance mechanisms to broad-spectrum antibiotics, including polymyxin. 
Thus, K. pneumoniae infection represents a growing public health concern. An increase in the colistin resistance rate has been reported among multidrug-resistant $K$. pneumoniae isolates, particularly among carbapenem-resistant K. pneumoniae (CR-KP) isolates. ${ }^{7}$ Outbreaks caused by carbapenem- and colistin-resistant isolates have also been reported in North America and Europe. ${ }^{7}$ Therefore, strategies to lower the dosage of polymyxin needed to clear resistant gram-negative infections are actively sought.

A complementary strategy for protecting existing drugs involves the use of antibiotic combinations and antibiotic adjuvants. ${ }^{8-11}$ Antibiotic combinations are beneficial because synergistic interactions are achieved through a variety of mechanisms, such as targeting distinct steps in common or linked biochemical pathways, enhancing uptake, and suppressing efflux. Synergistic effects can result in faster killing times or enhanced killing and thereby limit the opportunity for the emergence of resistant. ${ }^{12}$ In contrast to antibiotic combinations, antibiotic adjuvants show little or no antimicrobial activity alone. However, when combined with antibiotics, these adjuvants enhance antibiotic activity under specific conditions. ${ }^{13}$ The majority of polymyxin resistance mechanisms involve modifications that alter the lipopolysaccharide (LPS) structure and charge. ${ }^{14}$ One such modification is the addition of L-Ara4N to lipid A, which abrogates the initial charge-based interaction with the positively charged amino groups of polymyxin. ${ }^{14}$ ArnA, which is the first enzyme specific to the lipid A-Ara4N pathway, contains a dehydrogenase domain (ArnA_DH) that catalyzes the $\beta$-nicotinamide adenine dinucleotide $\left(\mathrm{NAD}^{+}\right.$)-dependent oxidative decarboxylation of UDP-glucuronic acid (UDP-GlcA) and a transformylase domain that formylates UDP-Ara4N. ${ }^{15-17}$ The essential role of ArnA in the addition of Ara4N to lipid A makes it an excellent potential target in polymyxin adjuvant design.

In view of the important role of ArnA_DH in lipid A-Ara4N synthesis, we launched a whole-cell screening program to search for inhibitors that target ArnA_DH using a dehydrogenase inhibitor library. The clinically used anti-alcoholism drug disulfiram (DSF), which acts as an aldehyde dehydrogenase inhibitor, ${ }^{12}$ was selected because it exhibited the strongest synergistic effect with PB against K. pneumoniae. Surprisingly, the enhancement effect of DSF on the antibacterial activity of PB does not appear to be achieved by the inhibition of dehydrogenases such as ArnA but by inhibition of the transcription level of genes associated with lipid A-Ara4N synthesis. Our study demonstrates the possibility of developing the combination of PB and DSF and reveals that DSF likely has a novel mechanism of action in bacteria.

\section{Methods}

\section{Compound Library and Screening Strategy}

A dehydrogenase inhibitor library was purchased from Selleck Chemicals (Houston, TX, USA). All bacterial strains used in this study were cultured in Luria-Bertani (LB) medium at $37^{\circ} \mathrm{C}$. PB (Sigma-Aldrich, USA) and inhibitors at twofold serial dilutions were prepared in $50 \mu \mathrm{L}$ of cation-adjusted Mueller-Hinton broth (CAMHB) in each well of 96-well plates. An aliquot of K. pneumoniae ATCC13883 (KP13883) culture $(50 \mu \mathrm{L})$ was then added to each well to obtain an $\mathrm{OD}_{600}$ of 0.006. The minimal inhibitory concentration (MIC) was defined as the lowest concentration that inhibited $90 \%$ of bacterial growth determined by the MTT assay (MCE, USA).

Combinations of compounds and PB were prepared in 96-well plates to obtain a final concentration of $100 \mu \mathrm{M}$ compound and $1 / 4 \times$ MIC PB in $50 \mu \mathrm{L}$ of $\mathrm{LB}$ per well. An aliquot of $\mathrm{KP} 13883$ culture $(50 \mu \mathrm{L})$ was then added to each well of the 96 well plate to obtain an $\mathrm{OD}_{600}$ of 0.006 . After overnight incubation at $37^{\circ} \mathrm{C}$, primary hits were identified as those showing $90 \%$ bacterial growth inhibition. Drug interactions were assessed through a checkerboard titration assay. The fractional inhibitory concentration (FIC) was calculated using the following formula: (MIC of drug A or B in combination)/(MIC of drug $\mathrm{A}$ or $\mathrm{B}$ alone). The fractional inhibitory concentration index (FICI) was the sum of the two FICs ( $\Sigma$ FIC). The combination was considered synergistic if $\Sigma$ FIC $\leq 0.5$, unrelated if $4 \geq \Sigma \mathrm{FIC}>0.5$, and antagonistic if $\Sigma \mathrm{FIC}>4 .{ }^{18}$

\section{Growth Curves of Bacteria}

A single colony of KP13883 was cultured in $3 \mathrm{~mL}$ of LB broth overnight at $37{ }^{\circ} \mathrm{C}$ with shaking at $230 \mathrm{rpm}$. The overnight cultures were then diluted 1:100 in fresh medium supplemented with $100 \mu \mathrm{M}$ DSF, $0.5 \mu \mathrm{g} / \mathrm{mL}$ PB or the combination of DSF with PB and cultured at $37^{\circ} \mathrm{C}$ with shaking at $230 \mathrm{rpm}$. The growth curves at $37^{\circ} \mathrm{C}$ were recorded by measuring the absorbance at a wavelength of $600 \mathrm{~nm}$ at intervals of 30 or $60 \mathrm{~min}$. 


\section{Protein Expression and Purification}

cDNA for full-length ArnA was chemically synthesized with the aim of optimizing its expression in Escherichia coli. The cDNA sequence was cloned into the protein expression vector pET28b. The plasmid was then transformed into the E. coli BL-21 strain for recombinant protein expression. The BL-21 strain carrying the aforementioned plasmid was grown in LB medium at $37{ }^{\circ} \mathrm{C}$ to an $\mathrm{OD}_{600}$ of 0.4 . The medium was then supplemented with $1 \mathrm{mM}$ isopropyl $\beta$ D-1-thiogalactopyranoside (IPTG), and the induced cells were grown at $37{ }^{\circ} \mathrm{C}$ for 4 hours.

To purify the recombinant proteins, the cells were harvested and lysed by ultrasonication. The supernatant of the lysed cells was loaded onto Ni-NTA columns (Qiagen, Germany), and the proteins were purified using a gel-filtration column (GE Healthcare, USA) with gel-filtration buffer (100 mM NaCl, $10 \mathrm{mM}$ Tris-HCl, $\mathrm{pH} 7.5$, and $1 \mathrm{mM}$ DTT). The protein concentration was determined using the Bradford method.

\section{Protein- or Cell-Based Effects of DSF on Enzyme Activity}

To assess its protein-based effects, KP13883 was cultured to an $\mathrm{OD}_{600}$ of 0.6 . The bacteria from $1 \mathrm{~mL}$ of culture were harvested (12,000 rpm, $5 \mathrm{~min})$, and total proteins were extracted using B-PER Bacterial Protein Extraction Reagent (Thermo Fisher, USA). Total protein $(0.1 \mathrm{mg})$ or $12 \mu \mathrm{g}$ of ArnA recombinant protein was treated in the presence or absence of DSF $(7.4 \mu \mathrm{g} / \mathrm{mL})$ and added to a reaction mixture containing $25 \mathrm{mM}$ Tris (pH 8.0), $5 \mathrm{mM}$ 2-mercaptoethanol, $0.2 \mathrm{mg} / \mathrm{mL}$ BSA, $10 \%$ glycerol, $100 \mathrm{mM} \mathrm{KCl,} 3 \mathrm{mM} \mathrm{NAD}^{+}$, and $1 \mathrm{mM} \mathrm{UDP-GlcA}$. The enzyme activity was measured by monitoring the increase in absorbance at $340 \mathrm{~nm}$ due to production of the reduced form of NAD (NADH) for $20 \mathrm{~min}$. All enzyme assays were performed at $37{ }^{\circ} \mathrm{C}$ in a final volume of $800 \mu \mathrm{L}$. The changes in NADH reflect UDP-GlcAspecific dehydrogenase activity.

For the assessment of cell-based effects, KP13883 cells were cultured to an $\mathrm{OD}_{600}$ of 0.6 in the presence or absence of $7.4 \mu \mathrm{g} / \mathrm{mL}$ DSF. The enzyme activity of $2 \mathrm{mg}$ of total protein was tested.

\section{Quantitative Real-Time PCR (qRT-PCR)}

Total RNA was extracted and purified using an RNAprep Pure Bacteria Kit (Tiangen, China). Reverse transcription and qPCR were performed using RT Master Mix for qPCR (MCE, USA) and SYBR Green qPCR Master Mix (MCE, USA) with 16S rRNA as the internal control. The primers used in the study are listed in Table S1.

\section{Isolation and Structural Analysis of Lipid A}

Mild-acid hydrolysis was used for lipid A extraction, as previously described. ${ }^{19}$ In brief, a single colony of KP13883 was cultured in $1 \mathrm{~mL}$ of LB broth overnight at $37{ }^{\circ} \mathrm{C}$ with shaking at $230 \mathrm{rpm}$. The bacteria were inoculated into fresh LB medium with or without DSF $(10 \mu \mathrm{g} / \mathrm{mL}), \mathrm{PB}(0.75 \mu \mathrm{g} / \mathrm{mL})$ or the combination of DSF $(10 \mu \mathrm{g} / \mathrm{mL})$ with PB $(0.75 \mu \mathrm{g} / \mathrm{mL})$ and shaken to an OD600 of 0.6-0.8. The bacteria were harvested by centrifugation at 13,000 rpm for $10 \mathrm{~min}$, and the cells were washed with PBS. An LPS extraction kit (iNtRON, Korea) was used for LPS extraction and purification. Ten milliliters of lysis buffer was added to each sample, and the mixture was vortexed vigorously. Each sample was added to $4 \mathrm{~mL}$ of chloroform, vortexed vigorously for $30 \mathrm{sec}$, and then incubated for $20 \mathrm{~min}$ at room temperature. The sample was centrifuged at $13,000 \mathrm{rpm}$ and $4{ }^{\circ} \mathrm{C}$ for $10 \mathrm{~min}$, and $8 \mathrm{~mL}$ of the supernatant was then transferred to a new $50-\mathrm{mL}$ centrifuge tube. Subsequently, $16 \mathrm{~mL}$ of purification buffer was added to the supernatant and mixed. The samples were incubated at $-20^{\circ} \mathrm{C}$ for $20 \mathrm{~min}$ and then centrifuged at $13,000 \mathrm{rpm}$ and $4{ }^{\circ} \mathrm{C}$ for $15 \mathrm{~min}$. The supernatant was removed, and the LPS pellet was washed with $20 \mathrm{~mL}$ of $70 \%$ ethanol and dried. Lipid A was hydrolyzed from LPS by incubation for $1 \mathrm{~h}$ at $100{ }^{\circ} \mathrm{C}$ in $5.4 \mathrm{~mL}$ of lysis buffer ( $\mathrm{pH} 4.5$ ) containing $50 \mathrm{mM}$ acetic acid-sodium acetate and $1 \%$ sodium dodecyl sulfate (SDS). The sample was cooled to room temperature, and chloroform $(6 \mathrm{~mL})$ and methanol $(6 \mathrm{~mL})$ were then added. The mixture was inverted, mixed and centrifuged at $2000 \times \mathrm{g}$ for $10 \mathrm{~min}$. The upper phase was collected, and $6 \mathrm{~mL}$ of the lower phase of the pre-equilibrated two-phase Bligh-Dyer mixture (chloroform:methanol:water; 2:2:1.8 v/v) was added. The sample was then centrifuged at $2000 \times \mathrm{g}$ for $10 \mathrm{~min}$. The lower phase was collected, and $22.8 \mathrm{~mL}$ of the upper phase of the pre-equilibrated two-phase BlighDyer mixture was added. The sample was then centrifuged at $2000 \times \mathrm{g}$ for $10 \mathrm{~min}$. The lower phase was collected, dried at 
room temperature, and stored at $-20{ }^{\circ} \mathrm{C}$. The structural analysis of lipid A was performed using a Q-Exactive Hybrid Quadrupole-Orbitrap Mass Spectrometer (Thermo Fisher) in the negative mode..$^{20,21}$

\section{Membrane Integrity Assay}

The KP13883 cultures were washed, resuspended in saline $(0.01 \mathrm{M})$, and standardized to an $\mathrm{OD}_{600}$ of 0.5 . Propidium iodide (PI) (Thermo Fisher Scientific) at a concentration of $10 \mathrm{nM}$ was added in the presence or absence of DSF or PB. After incubation at $37{ }^{\circ} \mathrm{C}$ for $30 \mathrm{~min}$, the fluorescence was measured with an excitation wavelength of $535 \mathrm{~nm}$ and an emission wavelength of $615 \mathrm{~nm}$.

\section{Bacterial Viability Assay}

The effect of DSF on bacterial viability was evaluated using a LIVE/DEAD BacLight Bacterial Viability Kit (Invitrogen, USA). The KP13883 cultures were washed, resuspended in saline $(0.01 \mathrm{M})$, and standardized to an $\mathrm{OD}_{600}$ of 0.1 . After incubation at $37{ }^{\circ} \mathrm{C}$ for $1 \mathrm{~h}$ in the presence of DSF $(100 \mu \mathrm{g} / \mathrm{mL})$ and $/$ or PB $(2 \mu \mathrm{g} / \mathrm{mL})$, the bacteria were harvested, washed, and resuspended in saline. SYTO9 $(1.67 \mathrm{mM}, 1.5 \mu \mathrm{L})$ and PI $(10 \mathrm{mM}, 1.5 \mu \mathrm{L})$ were added to each sample to obtain a final volume of $1 \mathrm{~mL}$ and incubated at room temperature in the dark for $15 \mathrm{~min}$. The stained bacteria were imaged using a confocal laser-scanning microscope (Leica TCS SP8).

\section{Mouse Pulmonary Infection Model}

A pneumonia model of clinical PB-resistant K. pneumoniae (P2418-1) in mice was used to test the antibacterial activity of the drug combination. Six- to eight-week-old female BALB/c mice raised under specific pathogen-free conditions were purchased from Hunan SJA Laboratory Animal Co., Ltd. The mice were intraperitoneally anesthetized with pentobarbital sodium $(75 \mathrm{mg} / \mathrm{kg})$ and inoculated with $1.0 \times 10^{7}$ colony-forming units (CFUs) of K. pneumoniae in $\mathrm{BALB} / \mathrm{c}$ noninvasive intratracheal instillation under direct vision. Two hours after infection, the drugs were administered via intraperitoneal injection; the treatments of PB and DSF alone were administered at doses of $0.2 \mathrm{mg} / \mathrm{kg}$ and $10 \mathrm{mg} / \mathrm{kg}$, respectively, and the combination treatments consisted of $0.2 \mathrm{mg} / \mathrm{kg}$ PB and 1,5 or $10 \mathrm{mg} / \mathrm{kg}$ DSF. The survival of the mice was observed for 7 days post-infection.

\section{Ethical Statement}

All animal care and use protocols in this study were performed in compliance with the Regulations for the Administration of Affairs Concerning Experimental Animals approved by the State Council of the People's Republic of China. All animal experiments in this study were approved by the Animal Ethical and Experimental Committee of the Army Military Medical University (Chongqing, approval No. 2011-04) in accordance with their rules and regulations.

\section{Data Availability}

We deposited the whole-genome sequencing (WGS) data of K. pneumonia clinical strains (P2445-4, AH1313-1, P2418-1, and P1291-19) in GenBank under BioProject PRJNA517992.

\section{Statistical Analyses}

Unpaired two-sided Student's $t$-test or one-way analysis of variance (ANOVA) was used for analyses of the transcription level, enzyme activity and lipid A modification level. Log-rank Mantel-Cox test was used for survival analysis. P $<0.05$ was considered to indicate statistical significance. All statistical processes were performed using GraphPad Prism 8.0 (GraphPad Software, Inc., San Diego, CA, USA).

\section{Results}

\section{Synergistic Combinations of Dehydrogenase Inhibitors and PB}

Taking the MIC of KP13883 $(2 \mu \mathrm{g} / \mathrm{mL})$ as a reference, we selected PB at a concentration of $1 / 4 \times \mathrm{MIC}(0.5 \mu \mathrm{g} / \mathrm{mL})$ for screening. At a concentration of $100 \mu \mathrm{M}$, no dehydrogenase inhibitors inhibited $90 \%$ of bacterial growth, but 5 promising 
drug combination pairs with $\Sigma$ FIC $\leq 0.5$ were identified by chequerboard titration (Table 1). Among these combinations, the strongest synergistic effect was observed with DSF and PB $(\Sigma F I C \leq 0.25)$. The growth curve showed that the combination of PB and DSF significantly inhibited bacterial growth (Supplementary Figure 1). This synergistic effect was also observed in clinical PB-resistant strains (Table 2). In addition, we found that diethyldithiocarbamate (DTC), the initial product of DSF metabolism in vivo, exerted no synergistic effect with PB (Table 2).

\section{Effect of DSF on ArnA Enzyme Activity}

Based on the synergistic effect of DSF on PB, we speculated that DSF may play a role by inhibiting the dehydrogenase activity of ArnA. However, the results of the spectrophotometric assay showed that ArnA activity was not affected by DSF (Figure 1A), which was not consistent with our hypothesis.

\section{Effect of DSF on the Transcription Level of Genes Associated with Lipid A Modification}

We then tested the effect of DSF on the transcription level of genes belonging to the arnBCADTEF operon (also known as pmrHFIJKLM), which encodes the enzymes responsible for the synthesis of Ara4 $\mathrm{N}$ precursors and its transfer to lipid A. ${ }^{14}$ The results showed that the transcription levels of $\operatorname{arn} A$, $\operatorname{arn} B, \operatorname{arn} C$, $\operatorname{arn} E$, $\operatorname{arn} F$ and $\operatorname{arn} T$ were significantly decreased after DSF treatment (Figure 1B). It is logical that the transcription of arn $A$ is synchronized with the transcription of other genes in the same operon, which suggests that the synergistic effect of DSF on PB is achieved by inhibiting the transcription of the arn operon rather than by inhibiting the dehydrogenase activity of ArnA.

\section{Effect of DSF on the UDP-GlcA-Specific Dehydrogenase Activity of Total Protein}

To identify the phase of action of DSF, we assessed the UDP-GlcA-specific dehydrogenase activity on total protein. UDP-GlcA-specific dehydrogenase activity was not affected by direct treatment of total protein with DSF. In contrast, the addition of DSF to the bacterial culture significantly reduced the enzyme activity of total protein, which indicated that DSF did not inhibit activity by directly acting on the enzyme (Figure 1C).

\section{Effect of DSF on the Lipid A Modifications of K. pneumoniae}

To investigate whether the inhibition of arn operon transcription could eventually prevent lipid A modification, we measured the proportion of modified lipid A from $K$. pneumoniae by LC-MS/MS. Different forms of modification, including the addition of Ara4N to the 1' or 4' phosphate (or both), were found (Figure 1D and Tables S2 and S3). The combinations of PB and DSF decreased the L-Ara4N-lipid A conjugates compared with the levels obtained with PB alone $(m / z 1841.24$ and $m / z$ 2063.47) (Figure 1D).

\section{Effect of DSF on Membrane Integrity}

As shown in Figure 2, we found that DSF had no effect on the integrity of the cell membrane. However, the combination of DSF and PB resulted in increased cell membrane disruption compared with that obtained with PB alone.

\section{DSF Enhanced the Activity of PB in vivo}

To evaluate the clinical value of the combination of DSF and PB, we selected a clinical strain of PB-resistant $K$. pneumoniae (P2418-1, MIC $=16 \mu \mathrm{g} / \mathrm{mL}$ ) for in vivo testing. Seven days after infection, the survival rates of the mice treated with PB $(0.2 \mathrm{mg} / \mathrm{kg}, 30 \%$ survival rate) or DSF (10 mg/kg, $0 \%$ survival rate) alone were lower than those of the mice treated with a combination therapy $(0.2 \mathrm{mg} / \mathrm{kg}$ PB +5 or $10 \mathrm{mg} / \mathrm{kg}$ DSF; $60 \%$ survival rates) (Figure 3 ). Consistent with the in vitro results, DSF showed no antibacterial activity in vivo at $10 \mathrm{mg} / \mathrm{kg}$. 
Table I Potency of Molecules in Dehydrogenase Inhibitor Libraries Alone or in Combination with Polymyxin B Against K. pneumoniae ATCCI3883

\begin{tabular}{|c|c|c|c|}
\hline Structure & Function & MIC $(\mu \mathrm{M})$ & $\Sigma$ FIC \\
\hline & $\begin{array}{l}\text { Noncompetitive, selective and reversible inosine monophosphate dehydrogenase I/ } \\
\text { II }\end{array}$ & $>100$ & $\leq 0.75$ \\
\hline & Phosphoglycerate dehydrogenase (PHGDH) inhibitor & $>100$ & $\leq 0.38$ \\
\hline & Aldehyde dehydrogenase (ALDHI) inhibitor used to treat chronic alcoholism & $>100$ & $\leq 0.25$ \\
\hline & Highly selective, effective mIDHI inhibitor with oral bioavailability & $>100$ & $\leq 0.38$ \\
\hline & $\begin{array}{l}\text { Dihydrodehydrogenase inhibitor that inhibits early homologous recombination in } \\
\text { double-strand break repair }\end{array}$ & $>100$ & $\leq 0.75$ \\
\hline & Unique product from licorice root with very high sweetness & $>100$ & $\leq 1$ \\
\hline & $\begin{array}{l}\text { Inhibitor of tetrahydrofolate dehydrogenase and antitumor antimetabolites with } \\
\text { immunosuppressive activity }\end{array}$ & $>100$ & $\leq 0.75$ \\
\hline & Potent and selective IDH2 RI40Q mutant inhibitor & $>100$ & $\leq 0.75$ \\
\hline
\end{tabular}

(Continued) 
Table I (Continued).

\begin{tabular}{|c|c|c|c|}
\hline Structure & Function & $\operatorname{MIC}(\mu \mathrm{M})$ & $\Sigma$ FIC \\
\hline & Selective inhibitor of I5-hydroxyprostaglandin dehydrogenase (I5-PGDH) & $>100$ & $\leq 0.28$ \\
\hline & New, potent and selective reversible inhibitor of IDH2 mutant enzymes & $>100$ & $\leq 0.75$ \\
\hline & Human dihydroorotate dehydrogenase (DHODH) inhibitor. & $>100$ & $\leq 0.50$ \\
\hline
\end{tabular}

Notes: $\sum \mathrm{FIC}$, sum of the fractional inhibitory concentration; $\sum \mathrm{FIC} \leq 0.5$ indicates synergism; $4 \geq \sum \mathrm{FIC}>0.5$ indicates indifference; and $\Sigma \mathrm{FIC}>4$ indicates antagonism.

Abbreviations: MIC, minimal inhibitory concentration; $\mathrm{FIC}$, fractional inhibitory concentration index.

Table 2 Potency of DSF Alone or in Combination with Polymyxin B Against Different Species

\begin{tabular}{|l|l|l|l|l|}
\hline \multirow{4}{*}{ DSF } & Species & Strains & MIC $(\mu \mathrm{M})$ & $\Sigma$ FIC \\
\cline { 2 - 5 } & Klebsiella pneumoniae & P2445-4 & $>100$ & $\leq 0.5$ \\
\cline { 2 - 5 } & Klebsiella pneumoniae & AHI3I3-I & $>100$ & $\leq 0.5$ \\
\cline { 2 - 5 } & Klebsiella pneumoniae & P24I8-I & $>100$ & $\leq 0.38$ \\
\cline { 2 - 5 } & Klebsiella pneumoniae & PI29I-19 & $>100$ & $\leq 0.25$ \\
\hline \multirow{2}{*}{ DTC } & Klebsiella pneumoniae & ATCCI3883 & $>100$ & $>1$ \\
\hline
\end{tabular}

Notes: $\sum$ FIC, sum of the fractional inhibitory concentration; $\sum$ FIC $\leq 0.5$ indicates synergism; $4 \geq \sum F I C>0.5$ indicates indifference; and $\Sigma F I C>4$ indicates antagonism.

Abbreviations: MIC, minimal inhibitory concentration; $\Sigma F I C$, fractional inhibitory concentration index; DSF, disulfiram; DTC, diethyldithiocarbamate.

\section{Discussion}

Because the primary interaction of polymyxins with the bacterial surface occurs via charge-based interactions with LPS, it is not surprising that the activity of polymyxins can be enhanced by promoting their binding to LPS. A good example of this phenomenon is that novobiocin enhances polymyxin activity by stimulating LPS transport. ${ }^{22}$ The controlled addition of positively charged residues, such as Ara4N, to LPS results in a reduced negative charge on the bacterial surface and therefore reduces the interaction between polymyxin and LPS. ${ }^{23}$ High-level polymyxin resistance due to the addition of Ara4N has been found in many bacterial clinical isolates. ${ }^{24-26}$ This finding suggests that inhibiting the modification of lipid A is an effective strategy for developing polymyxin adjuvants. ArnA dehydrogenase belongs to the short-chain dehydrogenase/reductase (SDR) family of enzymes that catalyze the NAD ${ }^{+}$-dependent oxidative decarboxylation of UDP-GlcA to UDP-4-keto-pentose, ${ }^{16,27}$ and this enzyme plays a crucial role in the pathway through which lipid A is modified with Ara4N, which leads to antimicrobial resistance in gram-negative bacteria. ${ }^{28}$ In this study, we aimed to identify compounds 

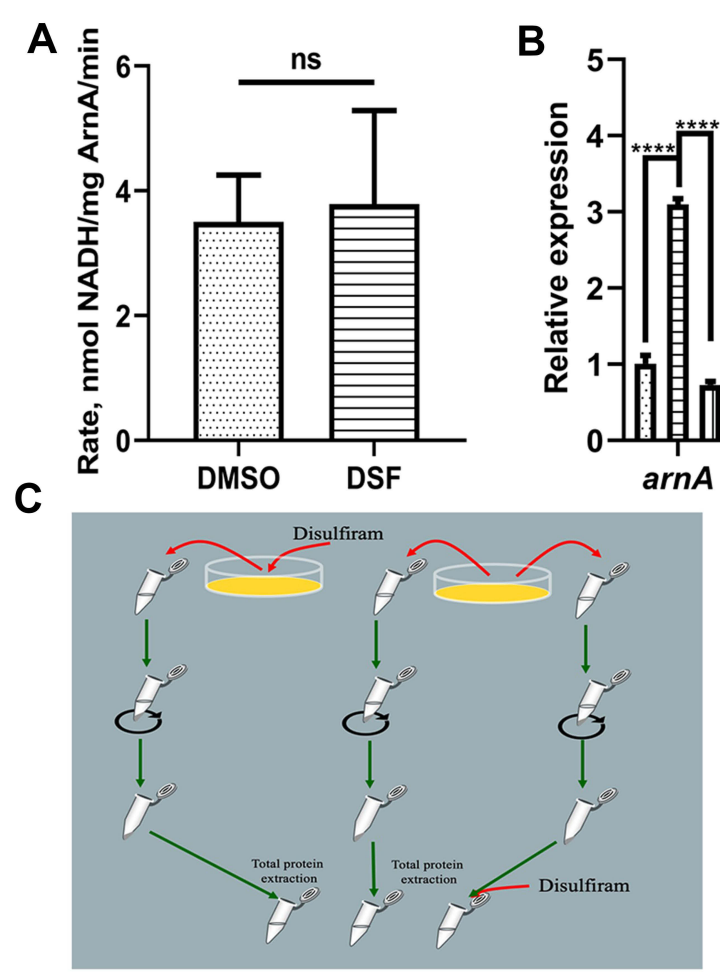

D

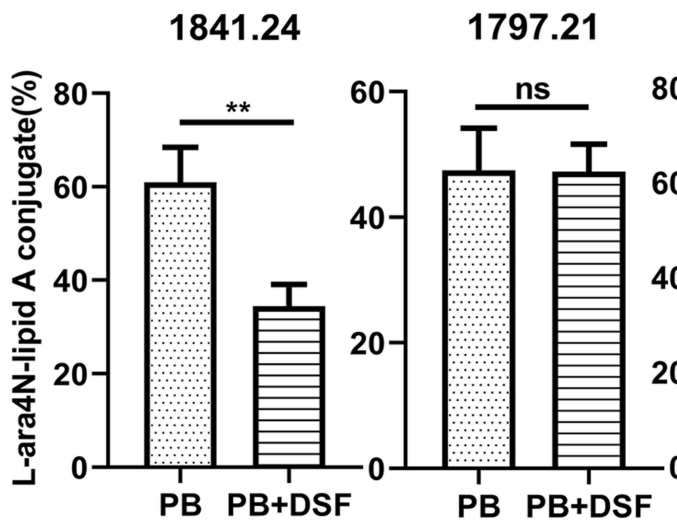

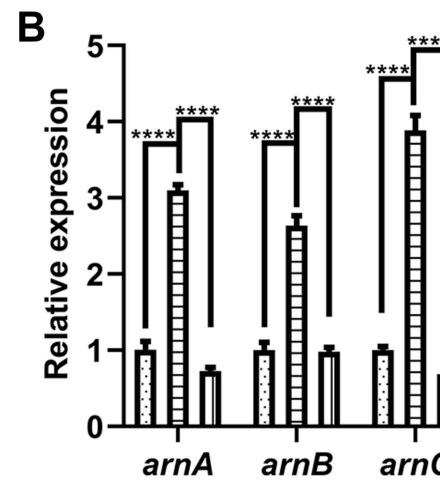
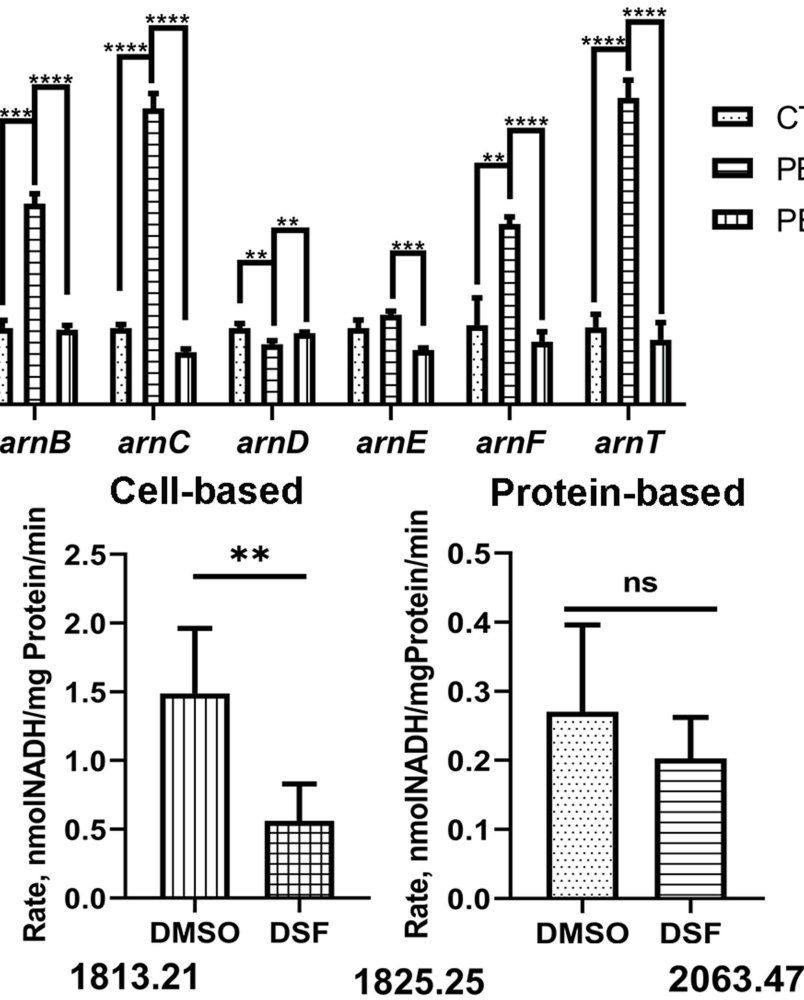

1825.25

2063.47
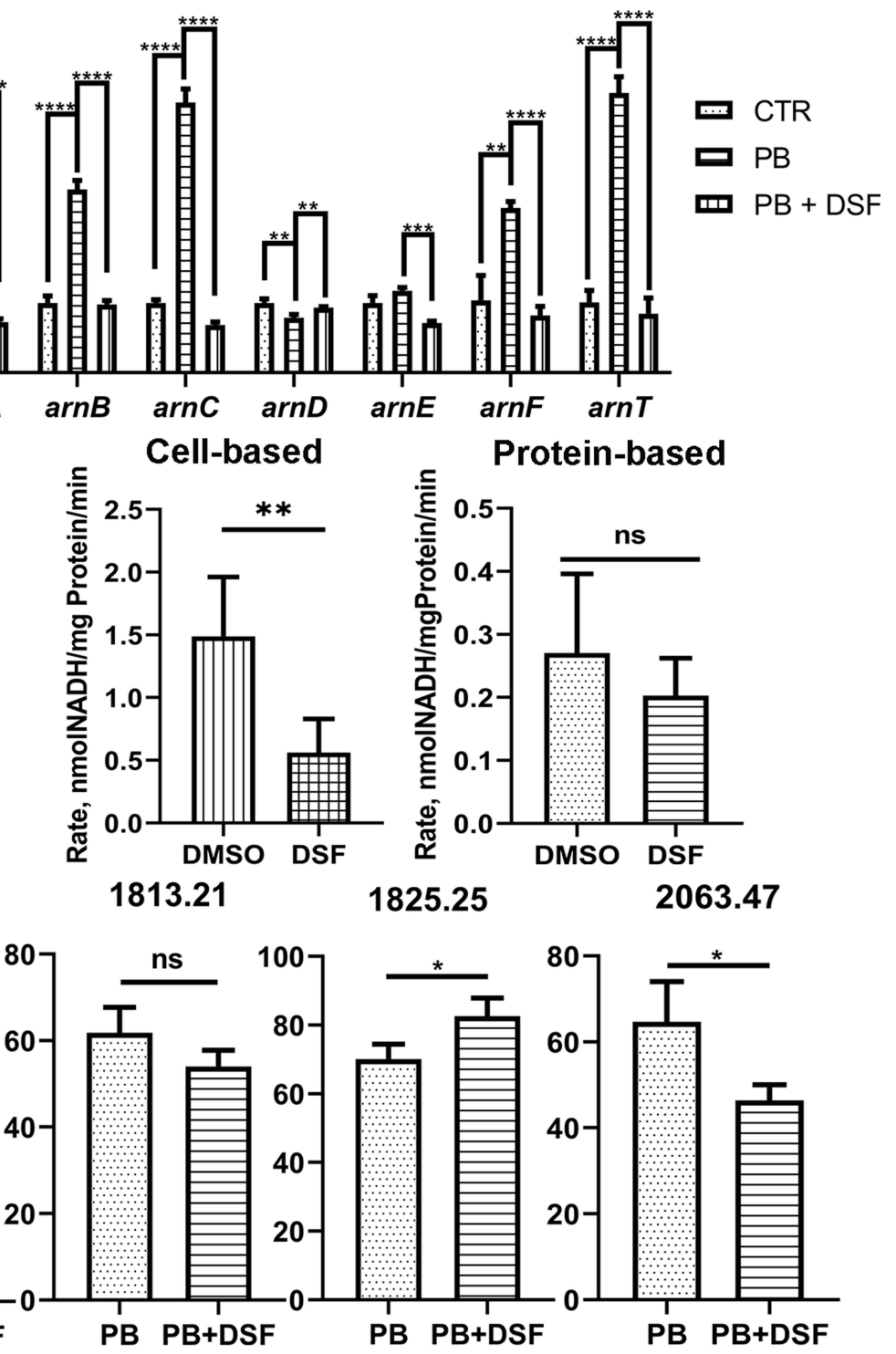

Figure I Inhibitory effect of DSF on the transcription level of genes in the arn operon and its inhibition of lipid A modification. (A) Effect of DSF on the UDP-GlcA-specific dehydrogenase activity of the recombinant ArnA protein. (B) Transcription levels of genes in the arn operon after treatment with PB alone or with the combination of PB and DSF. (C) Protein- and cell-based effects of DSF on the UDP-GlcA-specific dehydrogenase activity of total protein. A schematic of the sample addition operation is shown. DSF was added to the bacterial culture (cell-based) or total protein sample (protein-based). (D) The percent of the L-Ara4N-lipid A conjugate was determined based on LCMS/MS analysis. The reported structures of $K$. pneumoniae lipid A isolated from the strains included in this study are shown in Table $S 2$. $* P<0.05$, $* * P<0.01$, $* * * P<0.00 \mathrm{I}$, $* * * * P<0.0001$. The means are shown, and the error bars represent the means \pm standard errors of the mean (SEMs). Three biologically independent experiments were performed.

Abbreviation: ns, no significance.

from a dehydrogenase inhibitor library that could inhibit the function of ArnA_DH, which is a short-chain dehydrogenase. ${ }^{27}$ Surprisingly, although DSF exhibited PB enhancement activity, the related mechanism of action does not involve inhibition of dehydrogenase activity.

DSF, an irreversible inhibitor of aldehyde dehydrogenase, is a drug approved by the United States Food and Drug Administration (FDA) for alcoholism treatment. ${ }^{29}$ Due to its identified role in regulatory and stress-response pathways by directly targeting an adaptor of the p97 segregase NPL4, DSF is actively being repurposed for the treatment of cancer. ${ }^{30}$ Moreover, DSF was recently identified as a pyroptosis inhibitor that blocks gasdermin D pore formation. ${ }^{31}$ In this study, we describe a novel property of DSF as a transcription inhibitor in bacteria. DSF treatment downregulated the transcription of genes in the arn operon and inhibited the Ara4N modification of lipid A. Our 

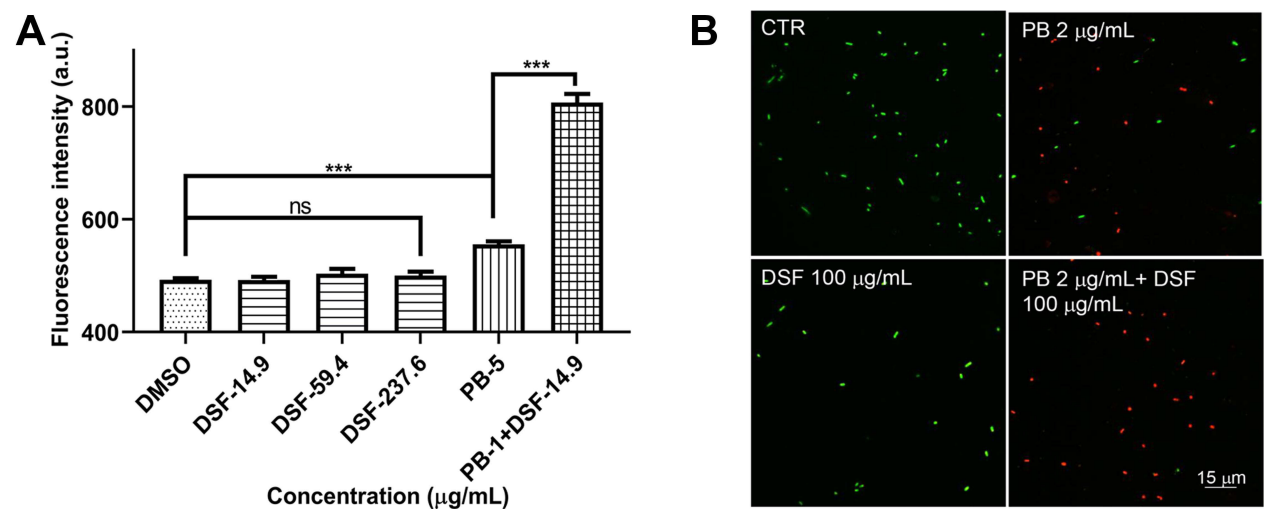

Figure 2 Effect of DSF on membrane integrity. (A) The integrity of the $K$. pneumoniae ATCCI3883 membrane was not affected by treatment with DSF alone (0-237.6 $\mu$ / $\mathrm{mL}$ ). DSF enhanced the disruption of the membrane integrity induced by PB. The membrane was probed with $10 \mathrm{nmol} / \mathrm{L} \mathrm{PI}$. ***P $<0.00 \mathrm{I}$. The means are shown, and the error bars represent the SEMs. The experiments were performed in triplicate. (B) Confocal images of $K$. pneumoniae ATCCI3883 after treatment with PB $(2 \mu g / \mathrm{mL})$ and/or DSF $(100 \mu \mathrm{g} / \mathrm{mL})$. The viable bacterial cells were stained green by SYTO9, and the dead cells were stained red by PI.

Abbreviations: a.u., arbitrary unit; ns, no significance.

\section{K. Pneumoniae P2418-1}

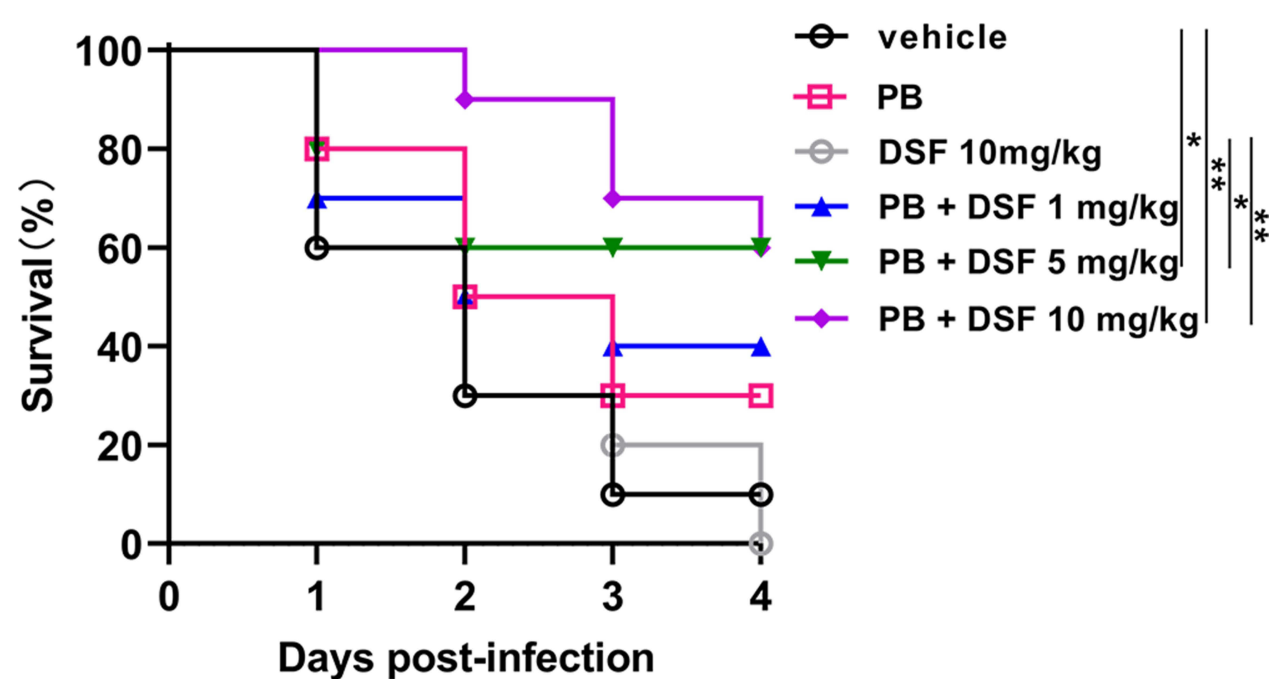

Figure 3 DSF enhances the activity of PB in a mouse infection model. The survival rates of mice with pulmonary infection $(n=10)$ are shown. Specifically, the survival rates of BALB/c mice $7 \mathrm{~d}$ after a single dose of $1.0 \times 10^{7}$ colony-forming units (CFUs) of PB-resistant $K$. pneumoniae $(\mathrm{P} 24 \mathrm{I} 8 \mathrm{I}, \mathrm{MIC}=16 \mu \mathrm{g} / \mathrm{mL}) \mathrm{were}$ determined. The survival rates increased after treatment with PB alone $(0.2 \mathrm{mg} / \mathrm{kg})$ or in combination with DSF $(\mathrm{I}, 5$ and $10 \mathrm{mg} / \mathrm{kg})$. $* P<0.05$, $* * P<0.01$.

study once again demonstrated that the identification of lipid A modification inhibitors is an effective method for identifying PB synergistic agents. However, as shown in Figure 1D, 1797.21, 1813.21 and 1825.25 Da did not show the expected difference between PB and PB combined with DSF, which might be due to the difference between the absolute amounts of different lipid A forms. In this study, we found the ratio between all detected lipid A and corresponding modified forms. In fact, 1797.21, 1813.21 and $1825.25 \mathrm{Da}$ is not high in terms of the overall absolute amount; thus, the low absolute amounts might be responsible for the unexpected result. In other words, because the total absolute amount is low, the difference might have a lower impact on the final drug-resistant phenotype of the bacteria.

Although we confirmed that DSF inhibits the transcription levels of genes belonging to the arn operon, the main limitation of our study is that the compound that directly interacts with DSF was not identified. The synergistic effect of DSF toward PB did not occur through its metabolites, such as DTC, which suggests that DSF did not act through the 
previously reported mechanism ${ }^{32}$ and that DSF may have as-yet unidentified targets in bacteria. In K. pneumoniae, the transcription level of the arn operon is mainly regulated by two-component systems (TCSs), such as PmrAB and PhoPQ. ${ }^{33,34}$ Moreover, regulators of PmrAB and PhoPQ, such as MgrB and CrrAB, and the intrinsic regulator RamA are also involved in regulating the modification of lipid. ${ }^{35,36}$ Therefore, all of the above-mentioned factors have potential as DSF targets and deserve further investigation.

The combination of DSF and PB was found to be effective against clinical PB-resistant strains. The safety profile of DSF, which has been used clinically for over six decades, underscores the promise of DSF and PB combination therapy for clinical application. ${ }^{37}$ Moreover, the animal experiments performed in this study verified that DSF enables PB to play an antibacterial role at low concentrations, which is significant for obtaining reduced side effects.

\section{Conclusion}

The combination of PB and DSF exhibited satisfactory antibacterial activity against gram-negative organisms, particularly K. pneumoniae, by inhibiting lipid A modification. This combination thus has good clinical application prospects, and its mechanism deserves further study.

\section{Abbreviations}

ArnA_DH, dehydrogenase domain; L-Ara4N, 4-amino-L-arabinose; PB, polymyxin B; MTT, 3-(4,5)-dimethylthiazol (-z-y1)-2,5-di-phenytetrazoliumromide; DSF, disulfiram; qRT-PCR, quantitative real-time polymerase chain reaction; CR-KP, carbapenem-resistant K. pneumoniae; LPS, lipopolysaccharide; UDP-GlcA, UDP-glucuronic acid; FIC, fractional inhibitory concentration; FICI, fractional inhibitory concentration index; LB, Luria-Bertani broth; CAMHB, cation-adjusted Mueller-Hinton broth; MIC, minimal inhibitory concentration; KP13883, K. pneumoniae ATCC13883; IPTG, isopropyl $\beta$-D-1-thiogalactopyranoside; NAD, $\beta$-nicotinamide adenine dinucleotide; NADH, reduced form of NAD; PI, propidium iodide; SDS, sodium dodecyl sulfonate; CFUs, colony-forming units; SEM, standard error of the mean; DTC, diethyldithiocarbamate; FDA, Food and Drug Administration; SDR, short-chain dehydrogenase/reductase; PEtN, phosphoethanolamine.

\section{Author Contributions}

WH conceived the research idea, guided the experiments and wrote the paper. JZ and QZ designed and performed the animal infection experiments. RC performed the screening, some in vitro assays and the data analyses. SL, CH, MZ and SC participated in part of the experiments. HY and MLZ read the draft of the manuscript and contributed to the editing of the manuscript. JW and YL provided the clinical strains. All authors contributed to the data analyses, drafted or revised the article, agreed to submit the manuscript to Infection and Drug Resistance, gave final approval of the version to be published, and agreed to be accountable for all aspects of the work.

\section{Funding}

This study was supported by the Shenzhen Key Medical Discipline Construction Fund (No. SZXK059, China), the Shenzhen Key Laboratory of Prevention and Treatment of Severe Infections (No. ZDSYS20200811142804014, China), the Guangdong Basic and Applied Basic Research Foundation (No. 2020B1515120066, China), and the National Nature Science Foundation of China (No. 82173859, China).

\section{Disclosure}

The authors declare no conflicts of interest associated with this work.

\section{References}

1. Rabanal F, Cajal Y. Recent advances and perspectives in the design and development of polymyxins. Nat Prod Rep. 2017;34(7):886-908. doi:10.1039/c7np00023e

2. Nation RL, Li J, Cars O, et al. Framework for optimisation of the clinical use of colistin and polymyxin B: the Prato polymyxin consensus. Lancet Infect Dis. 2015;15(2):225-234. doi:10.1016/S1473-3099(14)70850-3 
3. Kelesidis T, Falagas ME. The safety of polymyxin antibiotics. Expert Opin Drug Saf. 2015;14(11):1-15. doi:10.1517/14740338.2015.1088520

4. Justo JA, Bosso JA. Adverse reactions associated with systemic polymyxin therapy. J Pharmacother. 2015;35(1):28-33. doi:10.1002/phar.1493

5. Bialvaei AZ, Kafil HS. Colistin, mechanisms and prevalence of resistance. J Curr Med Res Opin. 2015;31(4):707-721. doi:10.1185/ 03007995.2015.1018989

6. Ah Y, Kim A, Lee J. Colistin resistance in Klebsiella pneumoniae. Int J Antimicrob Agents. 2014;44(1):8-15. doi:10.1016/j. ijantimicag.2014.02.016

7. Gales AC, Jones RN, Sader S. Contemporary activity of colistin and polymyxin B against a worldwide collection of Gram-negative pathogens: results from the SENTRY Antimicrobial Surveillance Program (2006-09). J Antimicrob Chemother. 2011;66(9):2070-2074. doi:10.1093/jac/ dkr239

8. Bernal P, Molina-Santiago C, Daddaoua A, et al. Antibiotic adjuvants: identification and clinical use. Microb Biotechnol. 2013;6:445-449. doi:10.1111/1751-7915.12044

9. Gill EE, Franco OL, Hancock RE. Antibiotic adjuvants: diverse strategies for controlling drug-resistant pathogens. Chem Biol Drug Des. 2015;85 (1):56-78. doi:10.1111/cbdd.12478

10. Kalan L, Wright GD. Antibiotic adjuvants: multicomponent anti-infective strategies. Expert Rev Mol Med. 2011;13(13):e5. doi:10.1017/ S1462399410001766

11. Wright G. Antibiotic adjuvants: rescuing antibiotics from resistance. Trends Microbiol. 2016;24:862-871. doi:10.1016/j.tim.2016.06.009

12. Worthington RJ. Combination approaches to combat multidrug-resistant bacteria. Trends Biotechnol. 2013;31(3):177-184. doi:10.1016/j. tibtech.2012.12.006

13. Brown D. Antibiotic resistance breakers: can repurposed drugs fill the antibiotic discovery void? Nat Rev Drug Discov. 2015;14(12):821-832. doi:10.1038/nrd4675

14. Gunn JS, Lim KB, Krueger J, et al. PmrA-PmrB-regulated genes necessary for 4-aminoarabinose lipid A modification and polymyxin resistance. Mol Microbiol. 1998;27(6):1171-1182. doi:10.1046/j.1365-2958

15. Raetz CR. Lipopolysaccharide endotoxins. Annu Rev Biochem. 2002;71:635-700. doi:10.1146/annurev.biochem.71.110601.135414

16. Gatzeva-Topalova PZ, May AP, Sousa MC. Crystal structure of Escherichia coli ArnA (PmrI) decarboxylase domain. A key enzyme for lipid A modification with 4-amino-4-deoxy-1-arabinose and polymyxin resistance. Biochemistry. 2004;43(42):13370-13379. doi:10.1021/bi048551f

17. Gatzeva-Topalova PZ, May AP, Sousa MC. Structure and mechanism of ArnA: conformational change implies ordered dehydrogenase mechanism in key enzyme for polymyxin resistance. Structure. 2005;13(6):929-942. doi:10.1016/j.str.2005.03.018

18. Odds F. Synergy, antagonism, and what the chequerboard puts between them. J Antimicrob Chemother. 2003;52(1):1-11. doi:10.1093/jac/dkg301

19. Zhou Z, Lin S, Cotter RJ, et al. Lipid A modifications characteristic of Salmonella typhimurium are induced by NH4VO3 in Escherichia coli K12. Detection of 4-amino-4-deoxy-L-arabinose, phosphoethanolamine and palmitate. J Biol Chem. 1999;274(26):18503-18514. doi:10.1074/ jbc.274.26.18503

20. Aye SM, Galani I, Han ML, et al. Lipid A profiling and metabolomics analysis of paired polymyxin-susceptible and -resistant MDR Klebsiella pneumoniae clinical isolates from the same patients before and after colistin treatment. J Antimicrob Chemother. 2020;75(10):2852-2863. doi:10.1093/jac/dkaa245

21. Henderson JC, O’Brien JP, Brodbelt JS, et al. Isolation and chemical characterization of lipid A from gram-negative bacteria. J Vis Exp. 2013;16 (79):e50623. doi:10.3791/50623

22. Mandler MD, Baidin V, Lee J, et al. Novobiocin enhances polymyxin activity by stimulating lipopolysaccharide transport. $J$ Am Chem Soc. 2018;140(22):6749-6753. doi:10.1021/jacs.8b02283

23. Moffatt JH, Harper M, Boyce JD. Mechanisms of polymyxin resistance. Adv Exp Med Biol. 2019;1145:55-71. doi:10.1007/978-3-030-16373-0_5

24. Moskowitz SM, Brannon MK, Dasgupta N, et al. PmrB mutations promote polymyxin resistance of Pseudomonas aeruginosa isolated from colistin-treated cystic fibrosis patients. Antimicrob Agents Chemother. 2012;56(2):1019-1030. doi:10.1128/AAC.05829-11

25. Nummila K, Kilpeläinen I, Zähringer U, et al. Lipopolysaccharides of polymyxin B-resistant mutants of Escherichia coli are extensively substituted by 2-aminoethyl pyrophosphate and contain aminoarabinose in lipid A. Mol Microbiol. 1995;16(2):271-278. doi:10.1111/j.1365-2958.1995. tb02299.x

26. Ernst RK, Yi EC, Guo L, et al. Specific lipopolysaccharide found in cystic fibrosis airway Pseudomonas aeruginosa. Science. 1999;286 (5444):1561-1565. doi:10.1126/science.286.5444.1561

27. Jörnvall H, Persson B, Krook M, et al. Short-chain dehydrogenases/reductases (SDR). Biochemistry. 1995;34(18):6003-6013. doi:10.1021/ bi00018a001

28. Breazeale SD, Ribeiro AA, McClerren AL, et al. A formyltransferase required for polymyxin resistance in Escherichia coli and the modification of lipid A with 4-amino-4-deoxy-L-arabinose. Identification and function oF UDP-4-deoxy-4-formamido-L-arabinose. J Biol Chem. 2005;280 (14):14154-14167. doi:10.1074/jbc.M414265200

29. Johansson B. A review of the pharmacokinetics and pharmacodynamics of disulfiram and its metabolites. Acta Psychiatr Scand Suppl. 1992;369:15-26. doi:10.1111/j.1600-0447.1992.tb03310.x

30. Skrott Z, Mistrik M, Andersen KK, et al. Alcohol-abuse drug disulfiram targets cancer via p97 segregase adaptor NPL4. Nature. 2017;552 (7684):194-199. doi:10.1038/nature25016

31. Hu JJ, Liu X, Xia S, et al. FDA-approved disulfiram inhibits pyroptosis by blocking gasdermin D pore formation. Nat Immunol. 2020;21 (7):736-745. doi:10.1038/s41590-020-0669-6

32. Shen ML, Johnson KL, Mays DC, et al. Determination of in vivo adducts of disulfiram with mitochondrial aldehyde dehydrogenase. Biochem Pharmacol. 2001;61(5):537-545. doi:10.1016/s0006-2952(00)00586-4

33. Cheng YH, Lin TL, Pan YJ, et al. Colistin resistance mechanisms in Klebsiella pneumoniae strains from Taiwan. Antimicrob Agents Chemother. 2015;59(5):2909-2913. doi:10.1128/AAC.04763-14

34. Wright MS, Suzuki Y, Jones MB, et al. Genomic and transcriptomic analyses of colistin-resistant clinical isolates of Klebsiella pneumoniae reveal multiple pathways of resistance. Antimicrob Agents Chemother. 2015;59(1):536-543. doi:10.1128/AAC.04037-14

35. De Majumdar S, Yu J, Fookes M, et al. Elucidation of the RamA regulon in Klebsiella pneumoniae reveals a role in LPS regulation. PLoS Pathog. 2015;11(1):e1004627. doi:10.1371/journal.ppat.1004627 
36. McConville TH, Annavajhala MK, Giddins MJ, et al. CrrB positively regulates high-level polymyxin resistance and virulence in Klebsiella pneumoniae. Cell Rep. 2020;33(4):108313. doi:10.1016/j.celrep.2020.108313

37. Cvek B. Nonprofit drugs as the salvation of the world's healthcare systems: the case of Antabuse (disulfiram). Drug Discov Today. 2012;17(910):409-412. doi:10.1016/j.drudis.2011.12.010

\section{Publish your work in this journal}

Infection and Drug Resistance is an international, peer-reviewed open-access journal that focuses on the optimal treatment of infection (bacterial, fungal and viral) and the development and institution of preventive strategies to minimize the development and spread of resistance. The journal is specifically concerned with the epidemiology of antibiotic resistance and the mechanisms of resistance development and diffusion in both hospitals and the community. The manuscript management system is completely online and includes a very quick and fair peer-review system, which is all easy to use. Visit http://www.dovepress.com/testimonials.php to read real quotes from published authors.

Submit your manuscript here: https://www.dovepress.com/infection-and-drug-resistance-journal 